\title{
Partial reinforcement effect following a shift from massed acquisition to spaced extinction
}

\author{
STEVEN J. HAGGBLOOM and ELIZABETH K. POND \\ Arkansas State University, State University, Arkansas 72467
}

\begin{abstract}
Rats received massed (5-min ITI) acquisition trials in a straight runway on either a PRF or a CRF schedule followed by massed or spaced (24-h ITI) extinction according to a 2 by 2 factorial design. PRF groups were more resistant to extinction than CRF groups at both ITIs. The PRF extinction effect was only slightly reduced by the shift from massed acquisition to spaced extinction. These results support the view that the behavioral effects of ITI shifts are asymmetrical. The importance of Trial 1 cues in relation to ITI shifts and spontaneous recovery are discussed.
\end{abstract}

Responding in extinction and the partial reinforcement extinction effect (PREE) are regulated by the behavioral control exercised by internal stimulus representations of nonreward (SN) (Amsel, 1958; Capaldi, 1966). The PREE is a particularly robust phenomenon. It survives changes in motivation, type of reward, and response requirement (Ross, 1964). However, a shift in intertrial interval (ITI) from widely spaced acquisition trials to highly massed extinction trials (S-M ITI shift) reduces resistance to extinction and attenuates or eliminates the PREE, indicating that $\mathrm{S}^{\mathrm{N}}$ dissipates over time (Amsel, Wong, \& Traupmann, 1971; Capaldi, Berg, \& Sparling, 1971; Capaldi \& Haggbloom, 1975).

Specifically, Amsel et al. (1971) and Capaldi et al. (1971) suggested that $S^{N}$ contains permanent as well as short-term components. Massed trials contain both components, whereas only the permanent components are present at spaced trials. Partial reinforcement (PRF) groups trained at widely spaced trials do not learn to respond in the presence of the short-term component of $\mathrm{S}^{\mathrm{N}}$, but they experience those stimuli during massed extinction. It is the introduction of new and unconditioned values of $\mathrm{SN}^{\mathrm{N}}$ into the stimulus complex controlling behavior following an S-M ITI shift that reduces resistance to extinction.

The model proposed by Amsel et al. (1971) and Capaldi et al. (1971) is asymmetrical and predicts that shifts from massed acquisition to spaced extinction (M-S ITI shift) would not disrupt behavior. On the other hand, the general finding has been that any change in ITI reduces resistance to extinction (Mackintosh, 1974). Moreover, Capaldi (Note 1) reported that a shift from highly massed trials in acquisition to 1 trial/day in

This experiment was supported by a faculty research grant from Arkansas State University. The authors wish to thank John K. Beadles for the use of laboratory space in the Department of Biology. Requests for reprints should be addressed to Steven J. Haggbloom, Department of Psychology, Arkansas State University, P.O. Box 2127, State University, Arkansas 72467. extinction reduced the PREE in one experiment and eliminated it in another.

It seems unlikely that the disruptive behavioral effects of an M-S ITI shift are due to the introduction of previously unexperienced values of $\mathrm{S}^{\mathrm{N}}$. But the effect could, in part, be due to generalization decrement brought about by the removal of the massed-trial component of $\mathrm{SN}^{\mathrm{N}}$ from the stimulus complex controlling behavior (Capaldi \& Morris, 1976). The other two possibilities derive from the fact that at a 24 -h ITI, extinction occurs in the presence of cues uniquely associated with the first trial of the day (Trial 1 stimuli, or ST1), for example, due to the 24-h cyclic processes of the rat and the fact that that trial does not follow in the wake of previous trials (Bolles, 1967; Capaldi \& Morris, 1974). First, responding would be only weakly conditioned to ST1 if acquisition involved a very few days of training affording little exposure to that stimulus. Second, the behavioral control exercised by $\mathrm{SN}$ in extinction is dependent upon the reestablishment of the stimulus complex present in acquisition (e.g., Capaldi \& Morris, 1976). During massed acquisition, $\mathrm{SN}^{\mathrm{N}}$ is conditioned in the presence of cues associated with massed trials (SMT). If extinction trials are separated by a 24-h ITI, the stimulus complex present in extinction, ST1, differs from that present in acquisition, SMT.

The behavioral control exercised by ST1 may play an important role, not just in regulating the effects of an M-S ITI shift on resistance to extinction, but in regulating spontaneous recovery as well. For example, the spontaneous recovery that usually occurs from the last of a series of within-day massed extinction trials to Trial 1 of the next day could be due in part to the fact that fewer extinction trials have been administered in the context of ST1 than in the context of SMT. Extinction of responding in a black runway has little or no effect on response strength in a white runway (Long, 1965). ST1 and SMT might be as different for the rat as black and white runways (Capaldi \& Morris, 1974). 
In the experiment reported here, PRF and CRF groups received massed acquisition. Half of each group then received massed extinction trials, and the other half received extinction trials at a $24-\mathrm{h}$ ITI.

\section{METHOD}

\section{Subjects}

The subjects were 32 male albino rats, $80-100$ days old, bred in the laboratory from Holtzman stock.

\section{Apparatus}

The apparatus was a straight black runway, $130 \mathrm{~cm}$ long $x 9 \mathrm{~cm}$ high and wide. The first $25 \mathrm{~cm}$ made up the startbox, and the last $25 \mathrm{~cm}$ constituted the goalbox. The startbox was separated from the runway by a manually operated guillotine door. The goalbox, separated from the runway in the same manner, contained an unpainted wooden goal cup. Start times were recorded from the opening of the startbox door, which triggered a $.01 \mathrm{sec}$ clock, to a point $30 \mathrm{~cm}$ into the runway. Run and goal times were measured over the next 40 and $30 \mathrm{~cm}$, respectively. The offset of the first clock and the operation of the run and goal clocks were controlled by photoelectric circuitry.

\section{Procedure}

Two weeks prior to the 1 st day of experimental training, each rat was placed on a deprivation schedule, consisting of $12 \mathrm{~g}$ of Purina Lab Chow, which continued throughout the experiment. On Days 12-14, the rats were handled in squads of four for $4 \mathrm{~min} /$ day and were fed eight reinforcement pellets in the home cage.

On Day 14, eight rats were randomly assigned to each of four groups defined by a factorial combination of PRF vs. CRF acquisition schedules and massed vs. spaced extinction ITIs. The massed ITI was 4-5 min; the spaced ITI was $24 \mathrm{~h}$. The four groups were designated PRF-M, CRF-M, PRF-S, and CRF-S.

Beginning on Day 15, each group received four massed acquisition trials per day for 6 days. On reinforced (R) trials, the goal cup contained eight 45-mg Noyes pellets; on nonreinforced $(\mathrm{N})$ trials, the rats were confined to the goalbox for 20 sec. Groups CRF-M and CRF-S were rewarded on every trial in acquisition; Groups PRF-M and PRF-S received the following schedule of $\mathrm{R}$ and $\mathrm{N}$ trials: RRNR, RNNR, RNRR, RRNR, RNNR, RNRR. The weight of food eaten in reinforcement pellets was subtracted from the daily ration of lab chow. Rats were run in squads of four, containing one rat from each group. The running order for each squad was held constant, and running order within squads was randomized daily.

Extinction trials began on Day 21 for all rats. Groups CRF-M and PRF-M received three massed extinction trials on each of 4 days of extinction. Groups CRF-S and PRF-S received 12 extinction trials at the rate of 1 trial/day.

\section{RESULTS}

All times were converted to speeds in centimeters per second, and all analyses were performed on these speeds. Only goal speeds, which were representative of all sections, are reported.

A 2 (schedules) by 2 (ITIs) by 4 (trials) analysis of variance (ANOVA) across all trials on the last day of acquisition revealed no differences among groups due to schedule $[F(1,28)=1.13]$ or ITI $(F<1)$ and no Schedule by ITI interaction $(\mathrm{F}<1)$. A 2 (schedule) by 2(ITIs) ANOVA on the first trial of that day also indicated no differences at the end of acquisition (all Fs $<1$ ).

Running speeds for the first 12 extinction trials (12 days for Groups PRF-S and CRF-S; 4 days for Groups PRF-M and CRF-M) are summarized in blocks of six trials in Figure 1. As can be seen in Figure 1, Groups PRF-S and PRF-M were more resistant to extinction than were Groups CRF-S and CRF-M. Differences between Groups PRF-M and CRF-M increased over blocks, with Group PRF-M being more resistant to extinction than all other groups at the end of extinction.

These observations were confirmed by a 2 (schedules) by 2 (ITIs) by 3 (trials) by 4 (trial blocks) ANOVA. There was a significant schedule of reward $[F(1,28)=$ $21.56, p<.001]$ effect, but no differences due to ITI $(p<1)$ and no ITI by Schedule interaction $(F<1)$. However, there was a significant ITI by Schedule by Trial Blocks interaction $[F(3,84)=2.76, p<.05]$. The simple interaction of Schedule by Trial Blocks at massed trials $[F(3,63)=4.36, p<.01]$ showed that Group CRF-M extinguished at a faster rate than Group PRF-M. A simple interaction of ITI by Trial Blocks at the PRF schedule was also significant $[F(3,63)=3.31, p<.05]$, supporting what can be seen in Figure 1, that Group PRF-S extinguished at a faster rate than Group PRF-M. The relationship between Groups PRF-S and CRF-S and Groups CRF-S and CRF-M did not change over trial blocks $[F(3,63)=2.03$ and $F<1$, respectively $]$.

Groups PRF-M and CRF-M ran faster on the first extinction trial of each day than on the last extinction trial of the preceding day. To evaluate the hypothesis that such between-day spontaneous recovery is regulated by the behavioral control exercised by ST1, Figure 2

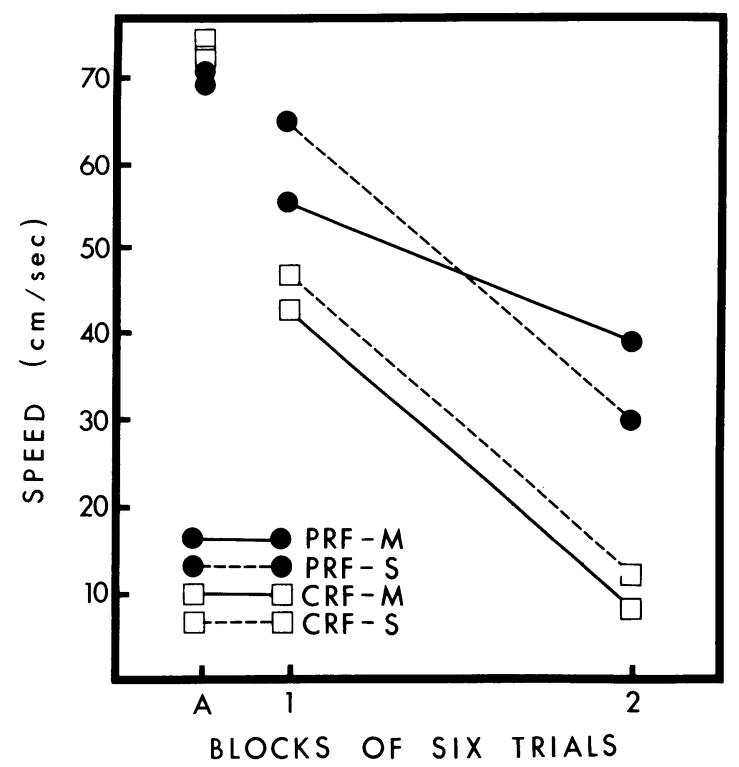

Figure 1. Mean goal speeds in blocks of six extinction trials for PRF and CRF groups given massed (M) or spaced (S) extinction. 


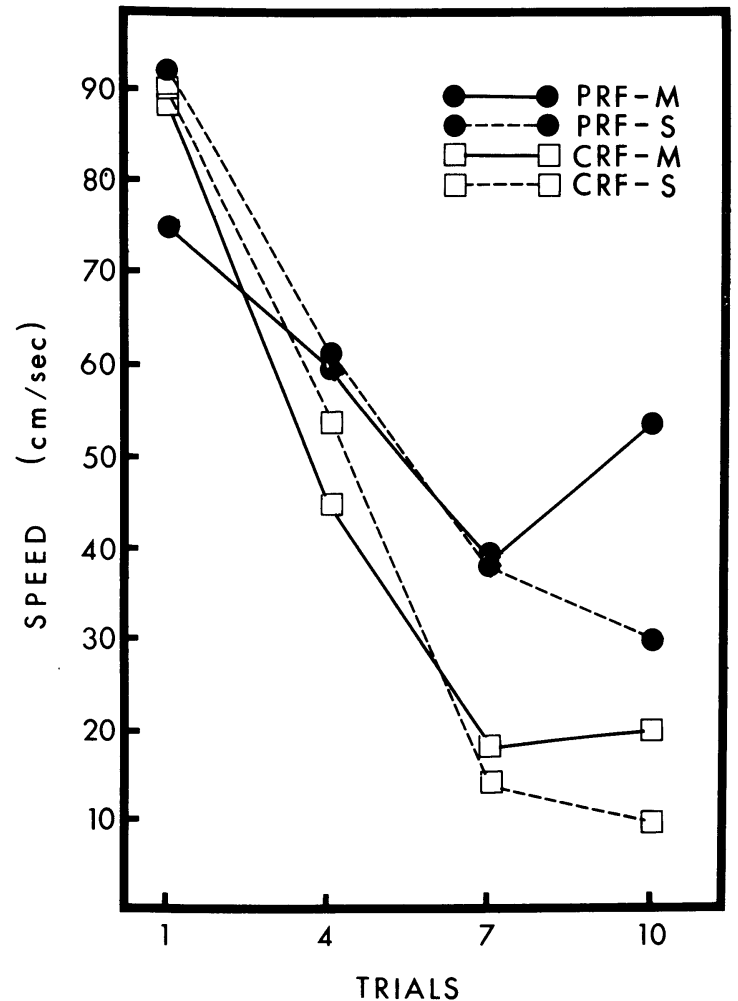

Figure 2. Mean goal speeds on Trials $1,4,7$, and 10 in extinction.

provides a comparison between all groups on extinction Trials $1,4,7$, and 10 . Trials 4,7 , and 10 are the first trials of the day for Groups PRF-M and CRF-M on Days 2, 3 and 4 of extinction. These trials are separated by equal numbers of intervening extinction trials for all groups.

As can be seen in Figure 2, responding in Groups PRF-M and CRF-M "spontaneously" recovered between days to essentially the same performance level as that in Groups PRF-S and CRF-S, respectively. A 2 (schedules) by 2 (ITIs) by 4 (trials) ANOVA applied to speeds on these trials showed that there were reliable differences due to schedule $[F(1,28)=5.25, p<.05]$, but no differences due to ITI $(F<1)$ and no ITI by Schedule interaction $(F<1)$. The fact that Groups PRF-M and CRF-M ran faster than Groups PRF-S and CRF-S, respectively, on Trial 10 contributed to a reliable ITI by Trials interaction $[F(3,84)=2.93, p<.05]$.

\section{DISCUSSION}

The present results are consistent with the hypothesis (Amsel et al., 1971; Capaldi et al., 1971) that the massed-trial value of $\mathrm{SN}^{\mathrm{N}}$ largely encompasses that of the spaced-trial value. This hypothesis predicts that an M-S ITI shift will produce a modest reduction in resistance to extinction compared with that produced by an S-M ITI shift. Capaldi (Note 1) reported that an
S-M ITI shift substantially reduced resistance to extinction after only 3 days of acquisition training and eliminated the PREE after only 4 days of training. Here, an M-S ITI shift following 6 days of acquisition training only slightly reduced the PREE. That the PREE was only slightly reduced here suggests that experience in responding to ST1 may be an important factor influencing resistance to extinction at a 24-h ITI. This view is consistent with the finding that an S-M ITI shift has a less disruptive effect on behavior in groups given experience responding to SMT (Capaldi \& Haggbloom, 1975).

This same argument could be extended to encompass the spontaneous recovery of responding from the last extinction trial of one day to the first extinction trial of the following day. In a massed extinction condition, the response undergoing extinction is that controlled by SMT. The first trial of each day, however, presents ST1 cues to which the rat has had limited extinction experience. As extinction days progress, and therefore the number of extinction trials to ST1 cues increases, spontaneous recovery is diminished. Viewed in this manner, the behavioral effects of ITI shifts appear to be due to processes similar to those that govern spontaneous recovery, as suggested by Mackintosh (1974).

\section{REFERENCE NOTE}

1. Capaldi, E. J. Reward schedule effects and shifts in intertrial interval from acquisition to extinction. Paper presented to the meeting of the Midwestern Psychological Association, Chicago, May 1974.

\section{REFERENCES}

Amsel, A. The role of frustrative nonreward in noncontinuous reward situations. Psychological Bulletin, 1958, 55, $102-119$.

Amsel, A., Wona, P. T. P., \& Traupmann, K. L. Short-term and long-term factors in extinction and durable persistence. Journal of Experimental Psychology, 1971, 90, 90-95.

Bolles, R. C. Theory of motivation. New York: Harper \& Row, 1967.

Capaldi, E. J. Partial reinforcement: A hypothesis of sequential effects. Psychological Review, 1966, 73, 459-477.

Capaldi, E. J., Berg, R. F., \& Sparling, D. L. Trial spacing and emotionality in the rat. Journal of Comparative Physiological Psychology, 1971, 76, 290-299.

Capaldi, E. J., \& Haggbloom, S. J. Response events as well as goal events as sources of animal memory. Animal Learning \& Behavior, 1975, 3, 1-10.

Capaldi, E. J., \& Morris, M. D. Reward schedule effects in extinction: Intertrial interval, memory, and memory retrieval. Learning and Motivation, 1974, 5, 473-483.

Capaldi, E. J., \& Morris, M. D. A role of stimulus compounds in eliciting responses: Relatively spaced extinction following massed acquisition. Animal Learning \& Behavior, 1976, 4, 113-117.

Long, J. B., McNamara, H. J., \& Gardner, J. O. Resistance to extinction after variable training as a function of multiple associations. Journal of Comparative Physiological Psychology, 1965, 60, 252-255.

Mackintosh, N.J. The psychology of animal learning. London: Academic Press, 1974.

Ross, R. R. Positive and negative partial reinforcement extinction effects carried through continuous reinforcement, changed motivation and changed response. Journal of Experimental Psychology, 1964, 68, 492-502.

(Received for publication September 5, 1981.) 